\title{
Acceptance of Hyperloop First empirical insights based on a representative study in the Netherlands
}

\section{November 2020}

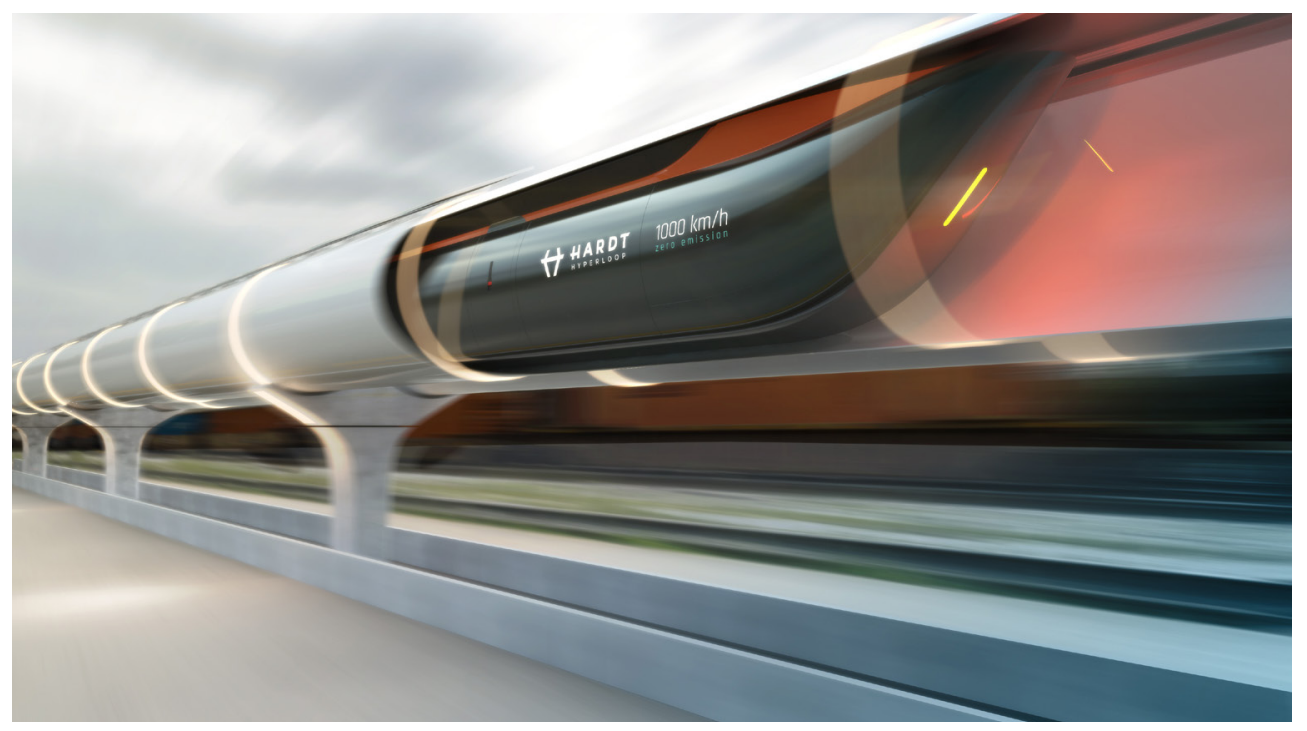

Image by Hardt Hyperloop

\section{Introduction}

Key Research Questions

\section{Would participants accept hyperloop and why or why not?}

\author{
How do different \\ levels of knowledge \\ affect the acceptance \\ of hyperloop?
}

Imagine travelling from Amsterdam to Berlin in one hour without boarding a plane and with no waiting time or emissions. These are the ambitious targets of hyperloop, a radical mobility innovation currently under development. The core technology of hyperloop is based on vehicles designed to transport people in low pressure tubes, accelerated by a magnetic levitation belt. Hyperloop technology presents opportunities for shortened travel time, a lower infrastructure footprint and an exceptionally low use of energy. Hardt Hyperloop, a technology company with headquarters located in the Netherlands, has developed a full-scale test facility that uses the core elements of hyperloop technology. Key factors for the successful introduction of hyperloop as a mass transportation solution include overcoming technical and economic challenges, as well as gaining acceptance among potential users. However, research on users' perspectives toward hyperloop technology remains scarce. The aim of this study is to generate a deeper understanding of user acceptance of hyperloop technology by identifying users' willingness to use this mode of transportation and the factors that support user acceptance or rejection of the new technology. The key challenge of this study is that most potential users are not currently aware of hyperloop technology and its development. Therefore, it is of particular interest to examine how differing levels of user knowledge about hyperloop affect its overall acceptance. 


\section{Demographics of the sample}

Gender

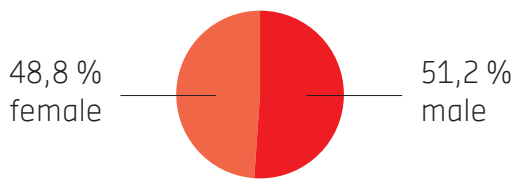

Age

\begin{tabular}{|ccc|}
\hline $21,4 \%$ & $52,5 \%$ & $26,1 \%$ \\
\hline under 30 & $30-50$ & over 50 \\
& & \\
\hline
\end{tabular}

Occupation

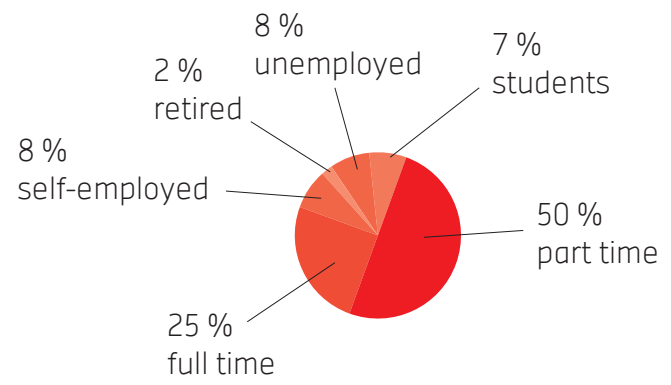

Income (in Euro)

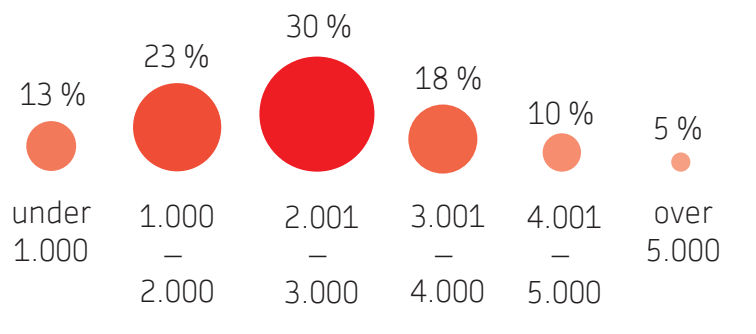

\section{Research approach and sample}

Data were collected from 8 July 2020 to 25 July 2020 via an online study conducted in the Netherlands, Germany and internationally. In the Netherlands, a representative sample of the overall population was utilized, based on a market research panel. The following results refer to the representative Dutch sample; other study results are available upon request. The sample size was 387 participants. The average participant age was 41 years, and participants ranged in age from 18-75 years. The sample consisted of $51.2 \%$ male and $48.4 \%$ female respondents. A wide variety of occupations, education levels and income groups were represented in the sample. More than $70 \%$ of respondents reported using a car at least once a week, whereas the majority of respondents reported using airplanes and (highspeed) trains less than once a month or never.

At the beginning of the questionnaire, hyperloop-related associations were collected from participants who had prior knowledge about the technology. Afterwards, all participants were provided with a short explanation of key facts about hyperloop. The questionnaire further covered a wide range of acceptance-related topics, including participants' reasons for using hyperloop, fears and concerns, willingness to use the technology and expectations of success.

The influence of prior participant knowledge about hyperloop on their acceptance of the technology was examined in two ways. The first analysis only considered participants' preexisting knowledge about hyperloop. In the second analysis, participants were provided with more hyperloop-specific information, including facts about travel speed, interior design and technical details. To examine the impact of the additional information, some acceptance-related topics (e.g. participant opinion and willingness to use the technology) were asked a second time after the additional information was provided. 


\section{Participants' associations with hyperloop are predominantly positive $(68.2 \%)$.}

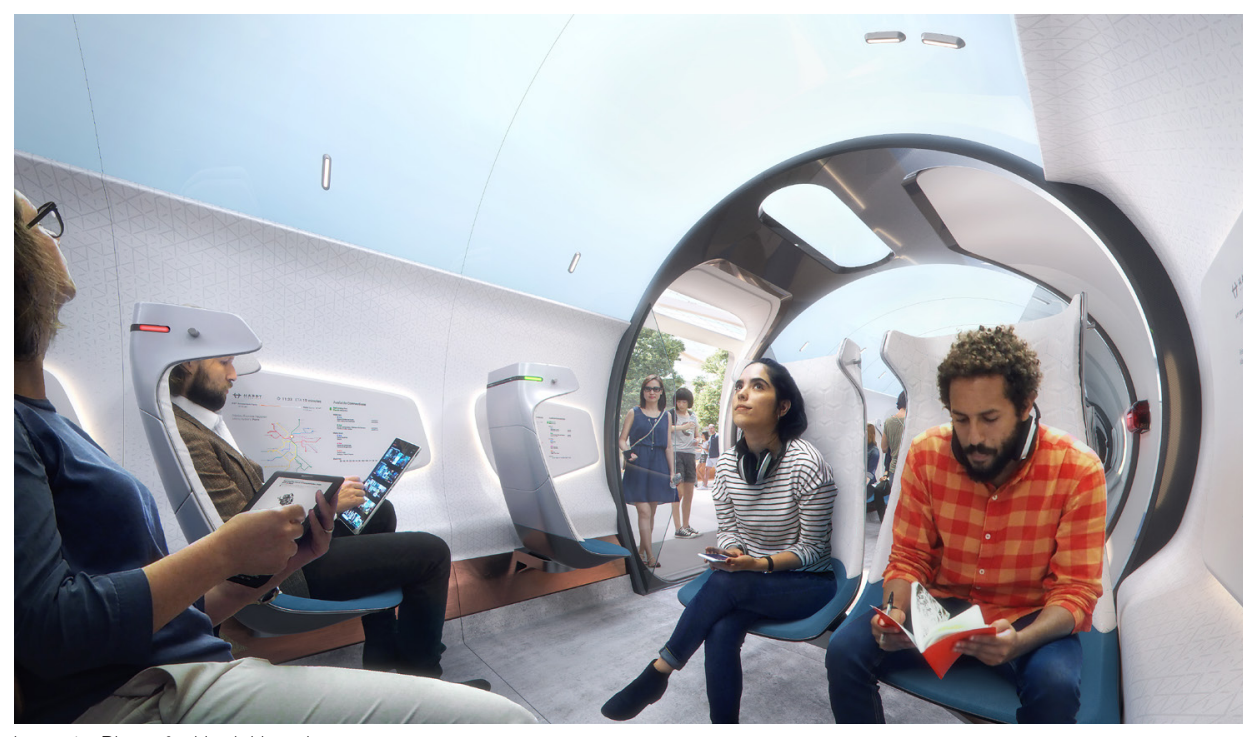

\section{Level of prior knowledge}

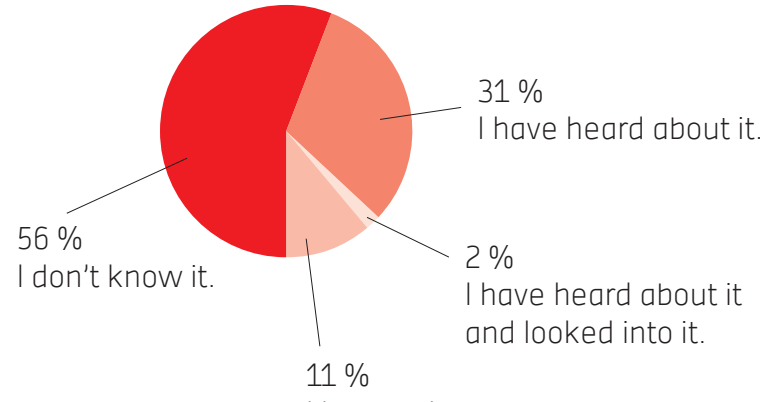

I know a lot about it.

\section{Hyperloop is expected to be fast, environmental friendly and to provide a quiet and comfortable ride.}

\section{Results}

\section{Prior knowledge and associations with hyperloop}

The majority of respondents (55.8\%) stated that they knew nothing about hyperloop technology prior to the study. Those who had heard of hyperloop beforehand only knew that it is a high-speed train. Therefore, hyperloop was positively associated with being fast. It was also associated with being modern, innovative and sustainable in a positive way. It is interesting to note that speed also held negative associations for some respondents. Other common negative associations included that hyperloop is utopian, expensive and dangerous. Overall, participants' associations with hyperloop were predominantly positive (68.2\%). Notably, respondents who reported a high level of prior knowledge about hyperloop had more positive associations with the technology than those who had only limited knowledge about hyperloop prior to the study. Men had significantly more knowledge about hyperloop than women, and overall, male participants had a more positive opinion than female participants regarding the technology. There was no observed difference between age groups with regard to prior knowledge or opinions about hyperloop.

\section{Reasons and barriers for using hyperloop}

As expected, the study data confirmed that the most important reason people would prefer to use hyperloop is the fast travel time. Other expected benefits that participants claimed would support their use of hyperloop included environmental friendliness, a quiet and comfortable ride and pricing that is comparable to other means of transportation. When asked to what extent they expected hyperloop to provide these benefits, respondents reported that they expected all but one of these benefits (pricing) would be provided by hyperloop. In other words, potential users expect hyperloop developers to deliver on their promises, but they assume that the technology will be priced higher than other means of transportation. Interestingly, social aspects such as the desire to impress others or to differentiate oneself from others by using hyperloop were not regarded as important. 


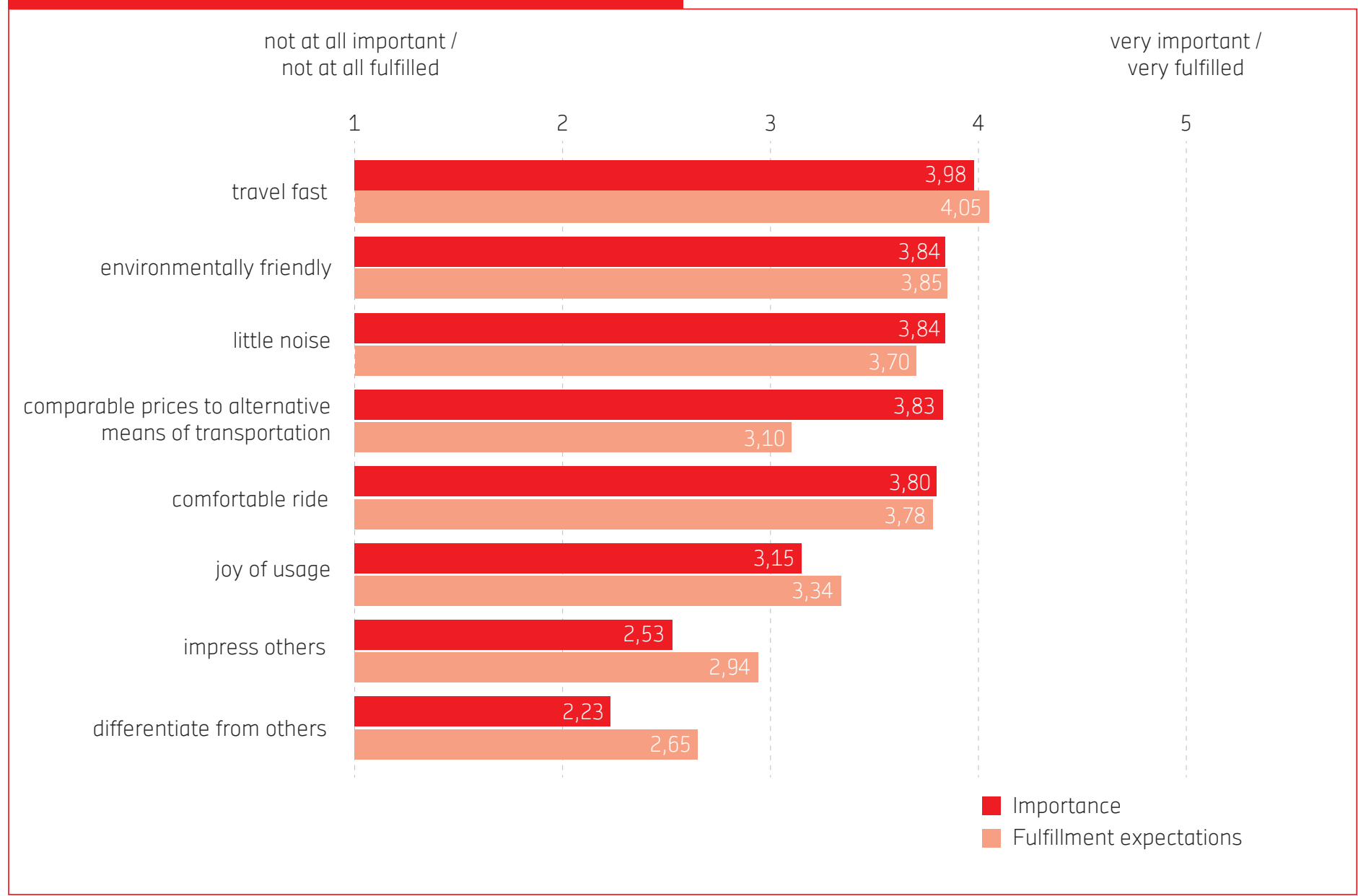

\section{I have concerns about using hyperloop}

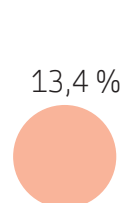

disagree

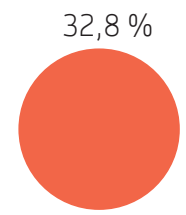

rather disagree

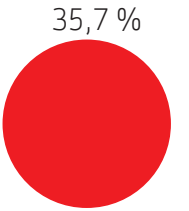

neither disagree nor agree
$14,7 \%$

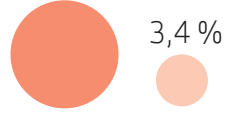

rather agree agree

\section{Half of the participants}

\section{(51.4\%) would use hyperloop in the future.}

Participants' negative associations regarding hyperloop indicate possible barriers to usage. Potential users reported that they were most concerned about the lack of windows and the lack of employees in the hyperloop vehicles. Other concerns included potential failure of the technology, as well as the low-pressure environment and the high travel speed. However, only a minority of all respondents $(18.1 \%)$ stated that they had concerns about hyperloop. In general, female respondents had more concerns than male respondents, and there were no differences between age groups.

\section{Willingness to use}

Participants were asked how likely it is that they would use hyperloop in the future. Despite their generally low level of current knowledge regarding the technology, half of the participants $(51.4 \%)$ reported that they would use hyperloop.

The previously mentioned reasons for using hyperloop, as well as fears and barriers to its use, impact participants' willingness to use the technology. Statistical methods were employed to examine which of the reasons for using hyperloop had the greatest impact on participants' willingness to use the technology. As expected, short travel time appeared to be the factor with the strongest impact on participants' willingness to use hyperloop. The second strongest predictor in the analysis was the joy of usage. In other words, a respondent's 


\section{How likely is it for you to use hyperloop in the future?}

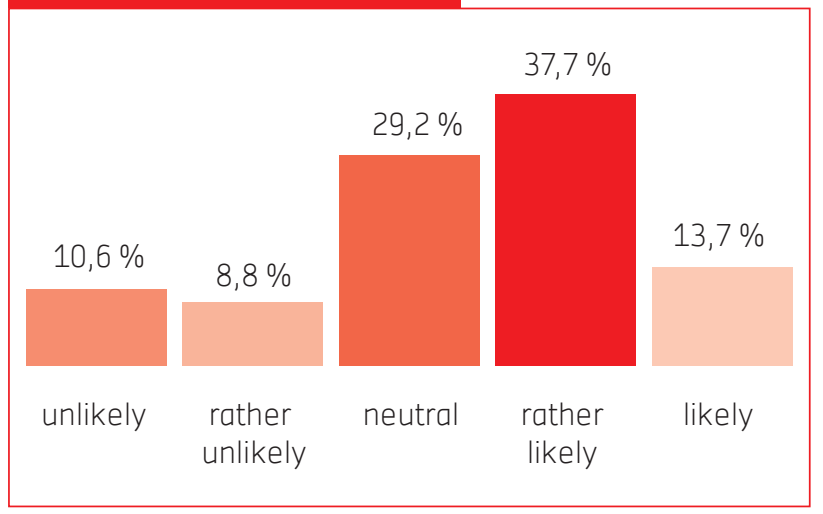

How does the level of prior knowledge affect the willingness to use? expectations of whether hyperloop will provide a joyful experience has a significant influence on their decision about whether to use the technology. Additionally, the results indicate that a high level of prior knowledge and information about hyperloop significantly increase participants' willingness to use the technology.

Male participants had more prior knowledge of the technology than their female counterparts. In accordance to this, male participants were more likely than female participants to report willingness to use hyperloop. Interestingly, age-specific differences were observed in participants' willingness to use the technology, despite the lack of age-related differences in prior knowledge about the technology. Specifically, younger participants reported that they were more likely to use hyperloop than older respondents. Participants under 30 are more likely to use hyperloop than participants older than 50. The data indicate that younger participants were more likely than older participants to expect the hyperloop travel experience to be fun, which may strongly influence their willingness to use the technology. In addition, participants who reported frequent use of trains, high-speed trains or airplanes were significantly more likely to report that they were willing to use hyperloop. This finding indicates that participant knowledge based on prior travel experience could lead to higher acceptance of the new technology.

willingness to use

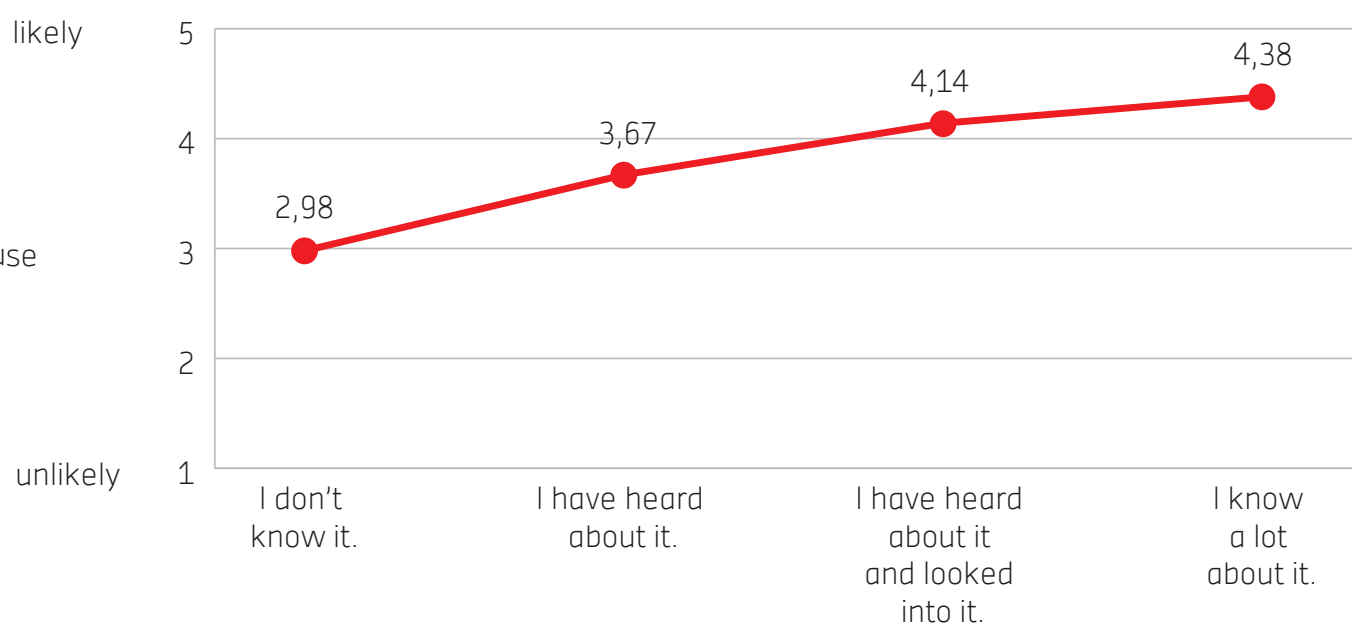

Respondents were asked whether they believed that hyperloop would be successful in their country. The majority of respondents believed that hyperloop would be succesful in the Netherlands, which indicates high societal acceptance. The data show that individual and societal acceptance are strongly correlated in that people who are likely to use hyperloop themselves consider others likely to use it as well. However, this finding also indicates that people who would not consider using hyperloop themselves may oppose the idea of building a hyperloop network in their country.

\section{Effects of provided knowledge}

As previously discussed, participants' prior level of knowledge regarding hyperloop had a positive influence on their acceptance of the technology. This study also examined to what extent provision of additional information about hyperloop would influence participants' 
acceptance of the technology. Respondents were provided with information about hyperloop, including facts about the speed and the technology itself, as well as images of the hyperloop interior. Respondents' opinions and expectations of the success of hyperloop technology remained on the same level after exposure to this information. Further, the results indicate that provision of additional information may increase participants' acceptance of hyperloop. This effect was particularly pronounced among respondents who had little prior knowledge about the technology, as well as respondents who reported few concerns about the technology.

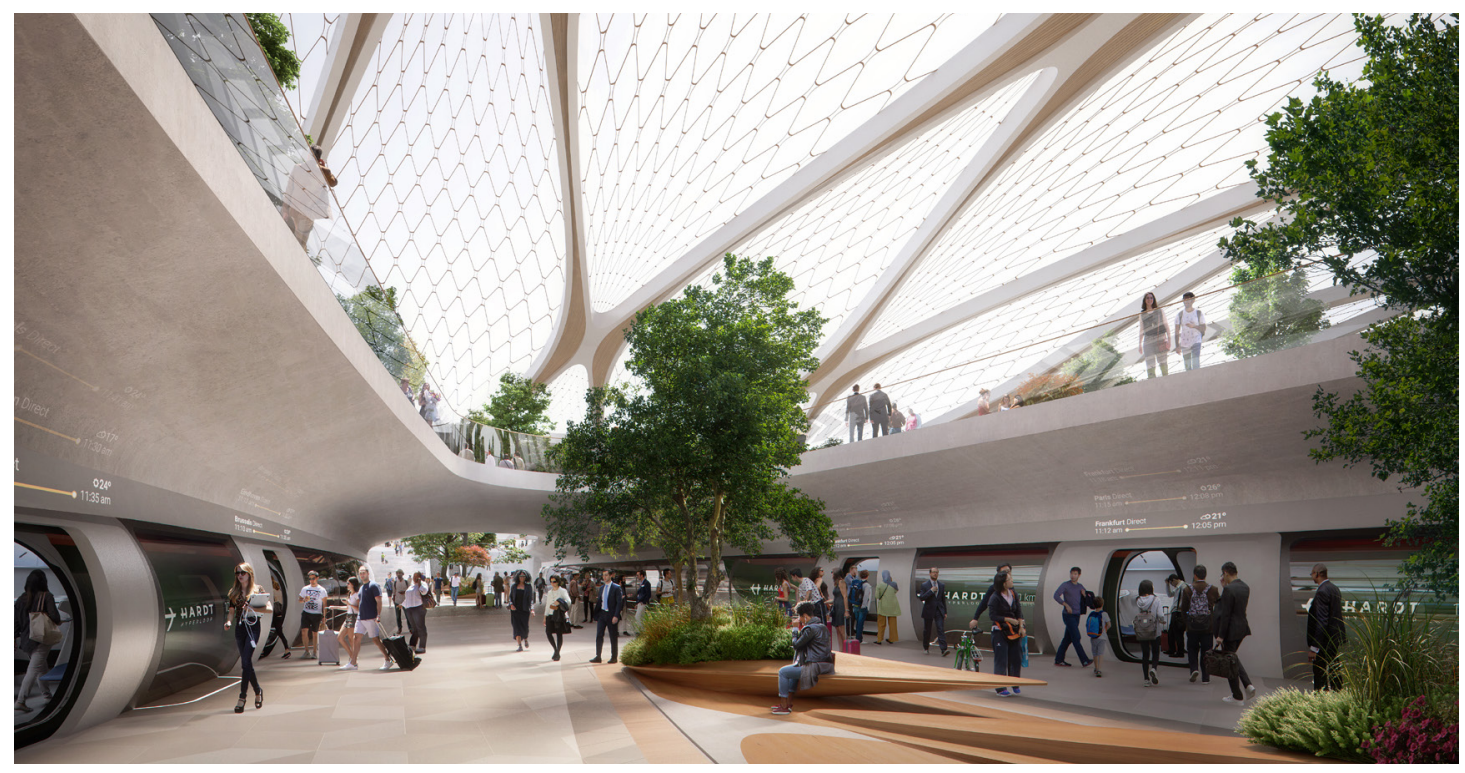

Image by UNStudio and Plomp for Hardt Hyperloop

\section{Conclusion}

In general, the study findings indicate a high level of acceptance of hyperloop among the Dutch population. Most participants had positive associations about the technology, and more than half of participants indicated that they would be willing to use hyperloop once it becomes available. These results are surprising because of the early stage of development of hyperloop technology and the resulting lack of knowledge about the technology among the population. Based on these findings, providing information about hyperloop could positively influence the attitude toward the technology, leading to even higher willingness to use hyperloop. However, increasing knowledge in the population may also lead to the opposite, because of new concerns that could be raised. Thus, the further development of the technology's overall acceptance in the society will depend on the way its core benefits and potential risks are publicly communicated by stakeholders. Stakeholder interesting in introducing hyperloop as future means of transport should put emphasis on the core perceived benefits of the technology (e.g. fast travel time and joy of use), as well as the main perceived concerns of the technology (e.g. lack of windows and lack of staff). The study results indicate that provision of additional information may help to overcome the objections of those with few concerns. To convince potential users with high levels of concerns to use the technology, other strategies will be needed, such as live experience of hyperloop. The potential impact of these strategies on acceptance of hyperloop technology will require further evaluation. 


\section{Limitations and outlook for further research}

Due to the early stage of development of hyperloop the knowledge of potential users is still limited and first-hand experience is lacking. In order to get a more realistic assessment of the attitudes regarding hyperloop, future studies could use Virtual Reality (VR) or Augmented Reality (AR) to simulate a hyperloop travel experience. Additionally, qualitative research could be utilized to better understand the fears and needs of potential users. Furthermore, since the present study only refers to a representative sample for the Netherlands, the acceptance of hyperloop in other European countries should be examined in future studies.

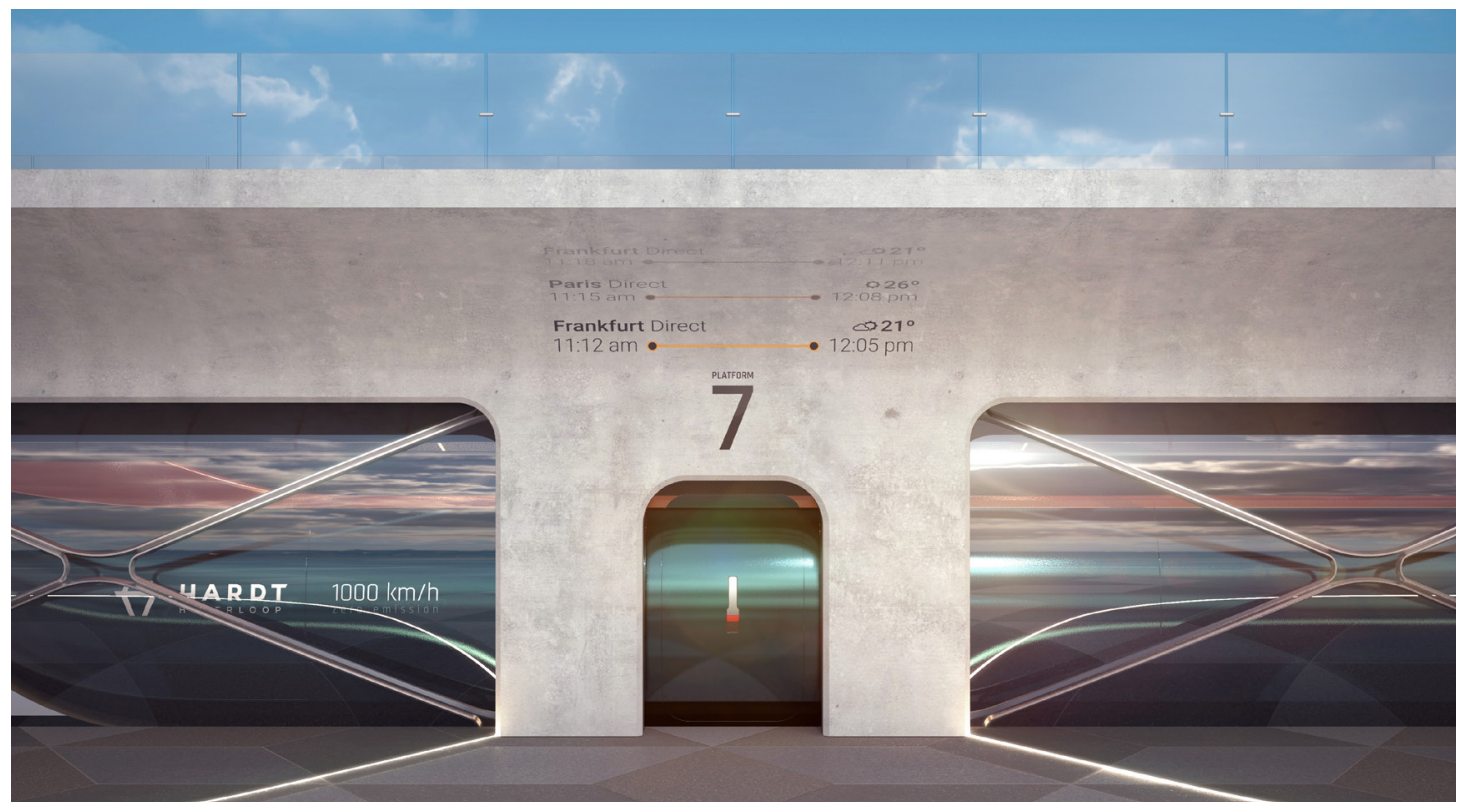

Image by Hardt Hyperloop

\section{Authors}

This research was conducted by AcceptanceLab, a research group at Hochschule für Technik Stuttgart - University of Applied Sciences, Schellingstraße 24 in 70174 Stuttgart, Germany.

Study Authors:

Patrick Planing, Anesa Aljovic, Jorina Hilser, Fee Furch

Contact details and further information can be found at www.acceptancelab.com

\section{Citation}

Planing, P., Aljovic, A., Hilser, J., \& Furch, F. (2020, November 3). Acceptance of Hyperloop - First empirical insights based on a representative study in the Netherlands. DOI: 10.31234/osf.io/7g2au

Available online at:

https://psyarxiv.com/7g2au/

\section{Acknowledgements}

This study was conducted in cooperation with Hardt Hyperloop, c/o Hardt B.V., The Netherlands.

This study was further supported by students of HFT Stuttgart - a University of Applied Science: Jaqueline Bueber, Surya de Benedetto, Tabea Dietrich, Leonie Friedrich, Philipp Müller, Selina Noeth, Emma Pütter, Nelly Schlien and Lisa Sulz. Many thanks for your support. 